\section{Efficient random variable generation: ratio of uniforms and polar rejection sampling}

\section{Luengo and L. Martino}

Monte Carlo techniques, which require the generation of samples from some target density, are often the only alternative for performing Bayesian inference. Two classic sampling techniques to draw independent samples are the ratio of uniforms (RoU) and rejection sampling (RS). An efficient sampling algorithm is proposed combining the RoU and polar RS (i.e. RS inside a sector of a circle using polar coordinates). Its efficiency is shown in drawing samples from truncated Cauchy and Gaussian random variables, which have many important applications in signal processing and communications.

Introduction: Bayesian inference has become very popular in signal processing and communications during the past few decades. Monte Carlo techniques, such as Markov chain Monte Carlo (MCMC) methods or particle filters, which are often necessary for their implementation, require the generation of samples from some target density [1]. Two classic sampling techniques, that are often used together, are the ratio of uniforms (RoU) and rejection sampling (RS). In this Letter we propose an efficient sampling algorithm combining the RoU and polar RS (i.e. RS inside a sector of a circle using polar coordinates), instead of the usual rectangular RS approach (i.e. RS inside a rectangular area using Cartesian coordinates), which can be inefficient in some cases. We show the efficiency of the algorithm in drawing samples from truncated Cauchy and Gaussian random variables (RVs), which have many important applications in signal processing and communications [2-4].

Ratio of uniforms and rejection sampling: RoU is a classic technique for generating samples from an arbitrary probability density function (PDF), $p_{0}(x)=k p(x)$ with $k>0$ [5]. Given a pair of independent RVs, $(u, v)$, uniformly distributed inside

$$
C_{p}=\{(u, v): 0 \leq u \leq \sqrt{p(v / u)}\}
$$

then $x=v / u$ is distributed exactly according to $p_{0}(x)$, i.e. $x \sim p_{0}(x)$. Hence, the RoU method allows us to draw samples from any PDF, as long as the resulting region $C_{p}$ is bounded, simply by generating a couple of independent uniform RVs.

Unfortunately, the efficiency and applicability of the RoU depends on the ability of generating uniform samples inside the region $C_{p}$, which is often not straightforward. The usual approach is embedding $C_{p}$ inside the rectangular region $R_{p}$ (Fig. $1 b$ ) and applying rectangular rejection sampling (RS). RS is another standard technique for generating samples from an arbitrary target $p_{0}(x)=k p(x) \mathrm{PDF}$, by using an alternative, simpler proposal PDF, $\pi(x)$, such that $p(x) / \pi(x) \leq L[5]$. RS works by generating samples from the proposal density, $w \sim \pi(x)$, accepting them when $z \leq p(w) /[L \pi(w)]$, with $z \sim U([0,1])$ uniformly distributed, and rejecting them otherwise.
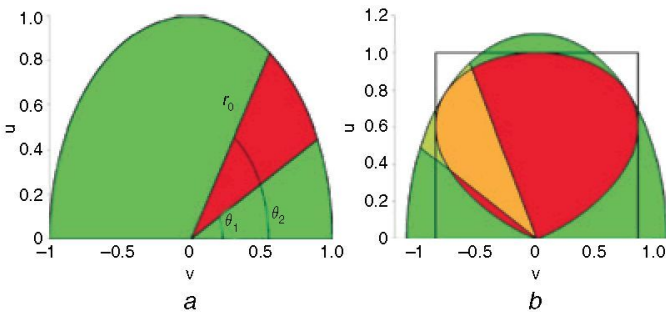

Fig. 1 Region $C_{p}$ and embedding regions $R_{p}$ and $S_{p}$

$a C_{p}=S_{p}$ for Cauchy and truncated Cauchy RVs

$b C_{p}, R_{p}$ and $S_{p}$ for a Gaussian and $S_{p}$ for a truncated Gaussian

The combination of the RoU and rectangular RS yields the following algorithm for generating each sample $x \sim p_{0}(x)$ : draw two independent uniform RVs, $(u, v)$, inside $R_{p}$; accept the $(u, v)$ pair if it belongs to $C_{p}$ (i.e. if the inequality in (1) is fulfilled), obtaining the generated $\mathrm{RV}$ as $x=v / u$; otherwise, discard $(u, v)$ and keep generating pairs of samples until $(u, v) \in C_{p}$. The key performance measure for this algorithm is the acceptance rate (i.e. the percentage of candidate samples accepted), which is given by $\Gamma_{R}=\left|C_{p}\right| /\left|R_{p}\right|$, where $\left|C_{p}\right|$ and $\left|R_{p}\right|$ denote the areas of the regions $C_{p}$ and $R_{p}$, respectively. Unfortunately, for many RVs of interest $\Gamma_{R}$ may be too low, leading to a large number of candidate samples being discarded. Therefore, alternative embedding areas which can be easily sampled, such as combinations of rectangles [6] or triangles [7], have been proposed to improve the acceptance rate.

RoU and polar RS: Another geometric area where uniform samples can be easily generated and that has not been exploited for RS is a sector of a circle, i.e. the region bounded by two radii of the circle and the arc of the circumference lying between them (Fig. 1a). Uniform sampling inside the sector delimited by angles $\theta_{1}$ and $\theta_{2}$ and radius $r_{0}$ can be achieved by drawing two independent samples from:

$$
\begin{gathered}
p_{e}(\theta)=\left\{\begin{array}{cc}
\frac{1}{\theta_{2}-\theta_{1}}, & \theta_{1} \leq \theta \leq \theta_{2} \\
0, & \text { otherwise }
\end{array}\right. \\
p_{R}(r)=\left\{\begin{array}{cc}
\frac{2 r}{r_{0}^{2}}, & 0 \leq r \leq r_{0} \\
0, & \text { otherwise }
\end{array}\right.
\end{gathered}
$$

Sampling from (2) only requires generating $u \sim U([0,1])$ and scaling it, obtaining the suitable angle as $\theta=\theta_{1}+\left(\theta_{2}-\theta_{1}\right) u$. Sampling from (3) is slightly more involved, requiring the generation of two independent $\mathrm{RVs}, v, w, \sim U([0,1])$. The desired radius is then:

$$
r=\left\{\begin{array}{cc}
r_{0} v, & w \leq v \\
r_{0}(1-v), & w>v
\end{array}\right.
$$

In this Letter we propose to combine the RoU and polar RS (i.e. RS using a sector of a circle, $S_{p}$, as the embedding region where we sample uniformly using polar coordinates) to improve the efficiency in the generation of some RVs, such as truncated Cauchy and Gaussian. The algorithm for generating a sample from the target PDF is the following:

1. Given a target PDF, $p_{0}(x)=k p(x)$ with $k>0$, find a sector of a circle, $S_{p}=\left\{(\theta, r): \theta_{1} \leq \theta \leq \theta_{2}, 0 \leq r \leq r_{0}\right\}$, such that $C_{p}$ is embedded inside $S_{p}$, i.e. $C_{p} \subseteq S_{p}$.

2. Draw a uniform sample pair inside $S_{p},(u, v)$, using polar coordinates as described by (2)-(4)

3. Accept it when $(u, v) \in C_{p}$, i.e. $0 \leq u \leq \sqrt{p(v / u)}$. In this case, the generated sample is $x=v / u$.

4. Otherwise, discard it and repeat steps 2-4.

Step 1 corresponds to the initialisation (i.e. it is performed only once), whereas the core of the algorithm (steps 2-4) only requires drawing three independent uniform RVs per generated sample. The acceptance rate in this case is $\Gamma_{S}=\left|C_{p}\right| /\left|S_{p}\right|$, with $\left|S_{p}\right|$ indicating the area of $S_{p}$.

Results: To show the applicability of the proposed algorithm, consider the generation of standard Cauchy RVs, $x \sim p(x)=1 /\left[\pi\left(1+x^{2}\right)\right]$, truncated or not. In this case, for the full Cauchy RV $C_{p}=\left\{(u, v): 0 \leq u^{2}+v^{2} \leq 1\right\}$ is the half-circle shown in Fig. $1 a$, whereas for the truncated Cauchy, $x \in\left[x_{1}, x_{2}\right], C_{p}$ is the sector shown also in Fig. $1 a$ with $r_{0}=1, \quad \theta_{1}=\pi / 2-\arctan \left(x_{1}\right)$ and $\theta_{2}=\pi / 2-\arctan \left(x_{2}\right)$. In both cases the proposed algorithm provides samples from the exact target PDF without any rejection (i.e. $\Gamma_{S}=1$ ). We remark that the inversion method [5], another popular sampling technique, can also be used here, but requires evaluating a tangent, thus leading to a higher computational cost than the proposed algorithm.

As a second example, we address the generation of standard Gaussian RVs, $x \sim p(x)=\frac{1}{\sqrt{2 \pi}} \exp \left(-x^{2} / 2\right)$. For a full Gaussian RV we obtain the region $C_{p}$ shown in Fig. $1 b$, which can be embedded inside a rectangle $R_{p}$ (with $\Gamma_{R} \approx 0.72$ ) or a circle $S_{p}$ (with $\Gamma_{S} \simeq 0.65$ ). Although the acceptance rate of rectangular RS is higher than the one for polar RS in this case, that is no longer true for truncated Gaussians. Fig. 2 shows the acceptance rate (averaged over 20000 runs) for different values of $x_{1}$ and $x_{2}$ and five RS algorithms: RS with constant upper bound (triangle-down marker), RS with a triangular-linear proposal (diamond marker), RS with a half-Gaussian proposal (triangle-up marker), RS with a truncated exponential PDF as proposal [8] (rectangular marker), and our polar RS method (circle marker). Our method provides the best acceptance rate when $0<x_{1}<1$ and $x_{2} \geq 3$ (Fig. 2b), and the second best for $x_{1}>1$ (Fig. $2 a$ ), only surpassed by the method from [8], which has a higher complexity. Note also that the 
first two approaches cannot be applied inside an infinite domain (i.e. when $x_{1} \rightarrow-\infty$ or $x_{2} \rightarrow-\infty$ ), whereas our approach is always feasible, regardless of the values of $x_{1}$ and $x_{2}$. Finally, we remark that the inverse cumulative function of a Gaussian is not known analytically. Hence, the commonly used inversion method [5] can only be applied approximately, whereas our method always provides samples from the exact target PDF.
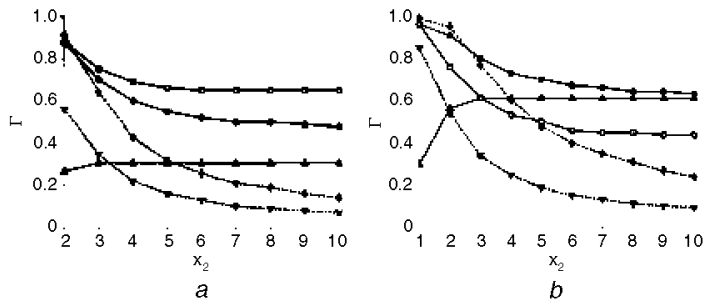

Fig. 2 Acceptance rates with different sampling methods

a $x_{1}=1, x_{2}$ variable

$b \quad x_{1}=0.5, x_{2}$ variable

Conclusions: An efficient algorithm for drawing samples from arbitrary univariate distributions based on the ratio of uniforms and a novel polar rejection sampling technique has been proposed. Its applicability and good performance has been demonstrated in the generation of full and truncated Cauchy and Gaussian RVs with the exact PDF and higher acceptance rates than other proposed methods. Adaptive versions of this method and extensions to multivariate PDFs can be easily implemented.

Acknowledgments: This work has been partly financed by the Spanish government, through the DEIPRO project (TEC2009-14504-C02-01) and the CONSOLIDER-INGENIO 2010 program (CSD2008-00010).

\section{References}

1 Ó Ruanaidh, J., and Fitzgerald, J.W.: 'Numerical Bayesian methods applied to signal processing' (Springer, New York, NY, USA, 1996)

2 Nadarajah, S.: 'Making the Cauchy work', Braz. J. Prob. Stat., 2011, 25, (1), pp. 99-120

3 Pitt, M., Chan, D., and Kohn, R.: 'Efficient Bayesian inference for Gaussian copula regression models', Biometrika, 2006, 93, (3), pp. 537-554

4 Zhu, Q.M., Dang, X.Y., Xu, D.Z., and Chen, X.M.: 'Highly efficient rejection method for generating Nakagami-m sequences', Electron. Lett., 2011, 47, (19), pp. 1100-1101

5 Devroye, L.: 'Non-uniform random variate generation' (Springer, New York, NY, USA, 1986)

6 Martino, L., and Miguez, J.: 'A rejection sampling scheme for posterior probability distributions via the ratio-of-uniforms method'. Proc. 18th EUSIPCO, Aalborg, Denmark, August 2010

7 Martino, L., and Miguez, J.: 'Two adaptive rejection sampling schemes for probability density functions log-convex tails', (arXiv:1111.4942v1, 21 November 2011)

8 Chopin, N.: 'Fast simulation of truncated Gaussian distributions', Stat. Comput., 2011, 21, pp. 275-288 